\title{
Highly Spectrally Efficient Discrete Multi-Tone Transmission over Step Index PMMA optical fibre for Short-Range Optical Communication
}

\author{
Flávio André N. Sampaio, Vinicius N. H. Silva, Luiz Anet Neto, Tadeu N. Ferreira, Andrés Pablo L. \\ Barbero, Ricardo Marques Ribeiro
}

\begin{abstract}
In this article we present high spectral efficiency DMT transmissions over $20 \mathrm{~m}$ of Step-Index polymethyl methacrylate optical fiber in the visible range. We demonstrated that using illumination light emitting diodes at 450, 520, 560 and $650 \mathrm{~nm}$ it is possible to achieve as high as $6.39 \mathrm{bits} / \mathrm{s} / \mathrm{Hz}$ for a preForward Error Correction with a target Bit Error Rate of $\mathbf{1 0}^{\mathbf{- 3}}$. The optical source at $560 \mathrm{~nm}$ was developed in our laboratory and then we demonstrated its usage as a new channel in WDM system.
\end{abstract}

Index Terms — Discrete Multi-Tone Transmission, Plastic Optical Fiber, Wavelength Division Multiplexing, Fluorescent Light Source

\section{INTRODUCTION}

$\mathrm{T}_{\mathrm{m}}^{\mathrm{h}}$ he worst disadvantages of step-index polymethyl methacrylate (SI-PMMA) optical fibers are its high attenuation profile and high modal dispersion limiting the distance and capacity of the communication link [1-2]. However, plastic optical fiber (POF) has several advantages such as low cost, immunity to electromagnetic interference (EMI) and easy handling. These characteristics put the POF as an excellent solution for short range communication. Indeed, this technology is already used in vehicles to provide communication between sensors, radio, GPS and in home networks to provide high communication data rates [3-4]. It is important to highlight that POF is not merely a promising solution; it is already in the market and being used in the development of new device and applications.

Several articles demonstrating data transmission in POF up to Gbps over 20, 50 and $100 \mathrm{~m}$ [5] have been published in the literature. These articles have shown the possibilities of communication systems and links with different types of modulation and multiplexing techniques, light sources and filters. Furthermore, laser diodes (LD), light emitting diodes (LED) and optical filters at different wavelengths became accessible in the market allowing improving transmission

A preliminary version of this article was presented in SBrT2017

Flávio Sampaio, Vinicius Silva, Tadeu Ferreira, Andrés Pablo Barbero, Ricardo Ribeiro are with Universidade Federal Fluminense, Telecom Engineering Dept., R. Passo da Pátria, 156, Niterói-RJ, 24210-240, Brazil (email: viniciusnhs@id.uff.br). Luiz Anet Neto is with Orange Labs Networks, Lannion, France, 22307 (e-mail: luiz.anetneto@ orange.com).

Digital Object Identifier: 10.14209/jcis.2018.25 capacity by means of wavelength division multiplexing (WDM), which is a consolidated technology for silica fibers [6]. However, the use of WDM in POF has not yet been widespread for data communication due to the high insertion losses caused by the light multiplexers and demultiplexers [7]. The very high single-wavelength bit-rates already demonstrated over POF prove that WDM for POF is still an open research field.

As a matter of fact, increasing the capacity of a WDM system can be achieved by either improving the single channel transmission, or adding new wavelength channel. Thus, since the attenuation profile in PMMA POF depends on its inherent characteristics, the solution to make higher bit-rates per wavelength is changing the modulation or multiplexing technique to mitigate the modal dispersion. On the one hand, Discrete Multitone (DMT) is a good candidate to increase the spectral efficiency [8]. On the other hand, a new wavelength optical carrier can be added to a WDM system if new optical sources and filters are developed. Many WDM techniques can have been reported in the literature recently [9-12]. However, none of them explore the yellow/orange transmission window (560-600 nm) because semiconductors compounds such as GaAsP, AlGaInP and GaP:N are not interesting materials allowing to develop fast, efficient and fiber-integrable sources at such window [7].

In this paper, we experimentally demonstrate high spectral efficiency transmissions using DMT in SI PMMA plastic optical fiber at 450, 520, 560 and $650 \mathrm{~nm}$. The $560 \mathrm{~nm}$ channel uses a light source developed in our lab [13]. It consists of a commercial fluorescent plastic optical fibre pumped by a green LED at $520 \mathrm{~nm}$. This device is lightweight, eye-safe, compact and we show that can be used as a new optical carrier to increase the capacity of WDM communication system. We have achieved data rates up to $128 \mathrm{Mbps}$ for a pre-forward error correction (FEC) target bit error rate (BER) of $10^{-3}$ over $20 \mathrm{~m}$ of SI-POF by means of simple direct intensity modulation with direct detection. Transmission spectral efficiencies as high as $6.39 \mathrm{bits} / \mathrm{s} / \mathrm{Hz}$ and aggregate data bit rates up to $455 \mathrm{Mbps}$ are 
demonstrated.

\section{PRELIMINARY AsSESSMENT OF THE EMITTERS}

Figure 1 shows the spectral characteristics of the optical sources used in the transmissions experiments. Their full width at half maximum (FWHM) are $34 \mathrm{~nm}, 40 \mathrm{~nm}, 31 \mathrm{~nm}$ and $22 \mathrm{~nm}$ for blue, green, yellow and red, respectively.

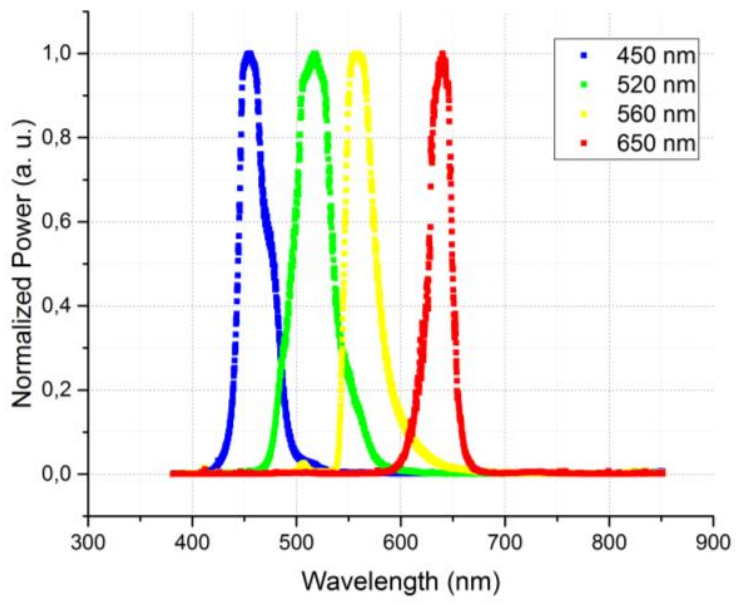

Figure 1 - Spectrum of the used LEDs.

In Figure 2 we show back-to-back frequency domain channel measurements obtained with a network analyzer. They allow us to measure the emitters' bandwidths at $-3 \mathrm{~dB}$, which will serve as reference values for choosing the DMT signal bandwidths. Respectively $24 \mathrm{MHz}, 17 \mathrm{MHz}, 10 \mathrm{MHz}$ and $9 \mathrm{MHz}$ are found for blue, green, yellow and red.

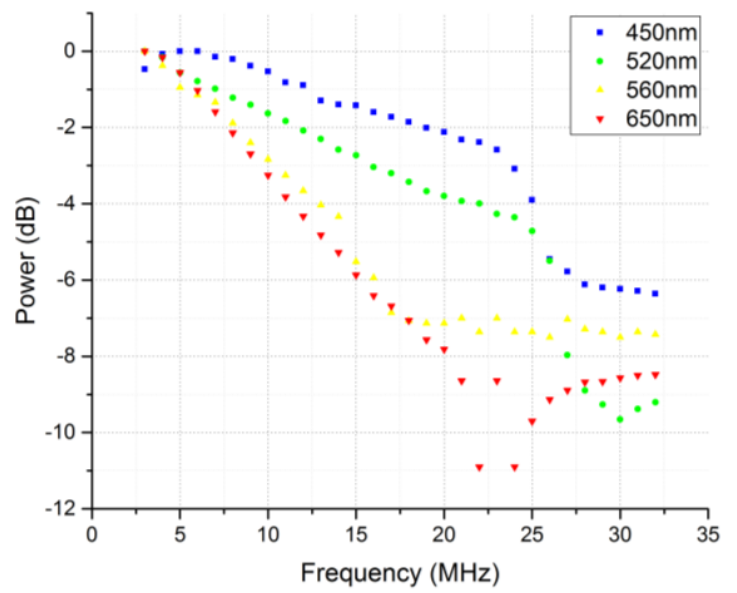

Figure 2 - Bandwidth characteristics the LEDs.

\section{DMT TRANSMISSION BLOCK}

The offline transmissions for all the optical carriers were done following the diagram in Figure 3. At the transmitter, a Pseudorandom Binary Sequence (PRBS) sequence is parallelized into lower bit-rate sequences. After that, they are mapped into $\mathrm{M}$-ary quadrature amplitude modulation (QAM) symbols $(\mathrm{M}=1,4,8, \ldots, 64)$ forming a subcarrier matrix (SM). In order to have a real discrete signal for direct intensity modulation and direct detection, the SM should be arranged to satisfy the Hermitian symmetry [14]. Then, each subcarrier into $\mathrm{SM}$ are multiplexed by applying the inverse fast Fourier transform (IFFT). A cyclic prefix (CP) is added to each timedomain DMT symbol to increased robustness against intersymbol interference (ISI) and to make the channel estimation, equalization and time synchronization as simple as possible. The signal is serialized $(\mathrm{P} / \mathrm{S})$ and then converted to analogic by a digital-to-analog converter (DAC). The electrical signal modulates the LEDs optical carriers, which are biased to operate in the linear region, at 450 (blue), 520 (green), 560 (yellow) and 650 (red) nm.
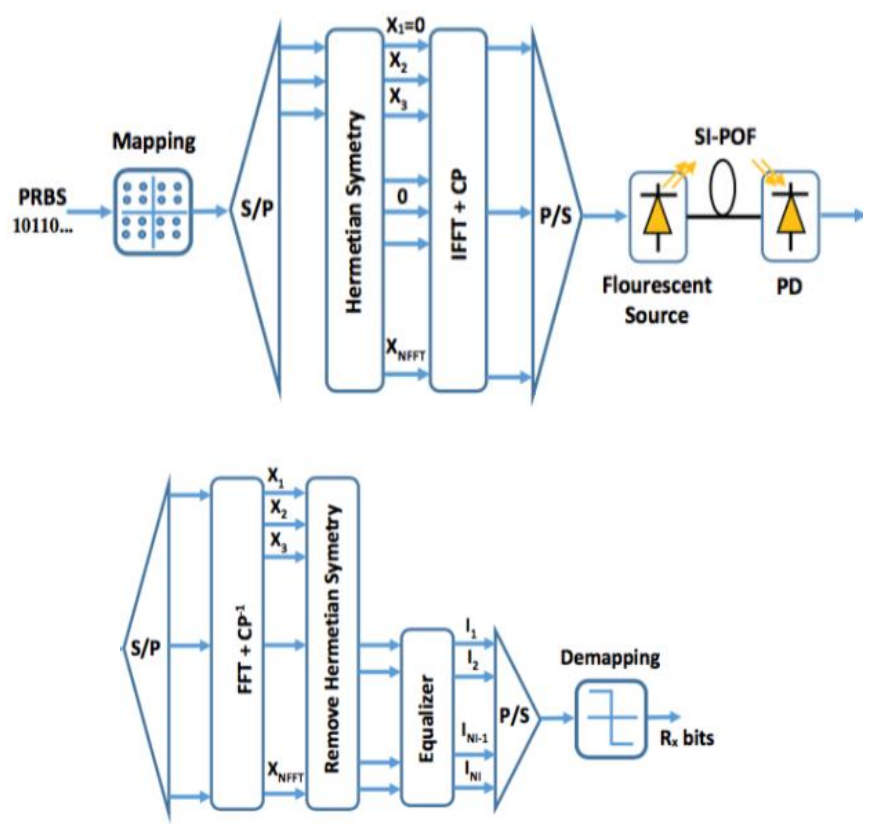

Figure 3 - DMT transmission block diagram.

The signal propagates through SI-POF and at the receiver side an analog-to-digital converter (ADC) digitizes the signal. The digital signal is then time synchronized and converted from serial to parallel before direct Fourier Transformation (FFT). The $\mathrm{CP}$ is removed, and the signal is frequency synchronized and equalized. Error vector magnitude (EVM) and signal to noise (SNR) are measured on the received symbols and finally the QAM symbols are demapped to provide actual BER measurements.

The bit and power optimization per subcarrier of the DMT signal is implemented in two steps. First, a probe signal is transmitted in which all subcarriers have the same power and are QPSK modulated. The first step allows measuring the signal-to-noise ratio (SNR) of the channel, which will be used by the rate-adaptive modified version of the Levin-Campello algorithm presented in [15-16]. This modified Levin-Campello [15-16] aims to reach a bit allocation policy closer to the channel capacity for each sub-carrier. A similar approach, with a distinct optimization problem and results, is found in [17]. The basic idea of this algorithm is to maximize the overall bitrate of the transmission as a function of a global signal power constraint and a target mean BER over all subcarriers. It is a 
greedy algorithm whose principle is to fulfill with more information (higher QAM modulation levels) the subcarriers with better SNR so that the overall bit-rate can be maximized. It then compensates the SNR fluctuations on subcarriers with the same modulation levels by changing the relative power coefficient of each subcarrier individually. In the second step of the transmission, a signal with the proper modulation and power coefficient per subcarrier is sent and a mostly constant BER over all subcarriers equal to the target BER is found.

The Levin-Campello algorithm itself has two phases. Starting from the initial two bits per QAM symbol (QPSK) on all subcarriers, the algorithm first "efficientizes" the bitdistribution. A bit-distribution is said to be efficient when there is no movement of a bit from one subcarrier to another that reduces the total energy of the DMT symbol. This replaces one bit-distribution with another closer to the efficient through single-information unit changes between subcarriers until no more changes can be done. An additional step is then necessary in order to adapt the total DMT symbol bit-rate with respect to the energy constraint. The second phase of the algorithm is known as "E-tight". E-tightness implies that no additional unit of information can be carried by the DMT signal without violating the total energy constraint.

\section{EXPERIMENTAL SETUP}

The experimental setup (Figure 4) consists of personal computer (PC) that performs DMT modulation/demodulation, an arbitrary function generator (Tektronik AFG3251), which works as a DAC, a bias-T (Mini-Circuits ZG85-12G+), a DC source, illumination LEDs (450, 520 and $650 \mathrm{~nm}$ ) from DieMount, a fluorescent optical source $(560 \mathrm{~nm})$ [13], $20 \mathrm{~m}$ of SI-POF, a photodetector (Thorlabs PDA10A) and an oscilloscope (Rohde\&Schwarz RTO 1002) to digitize the received signals.

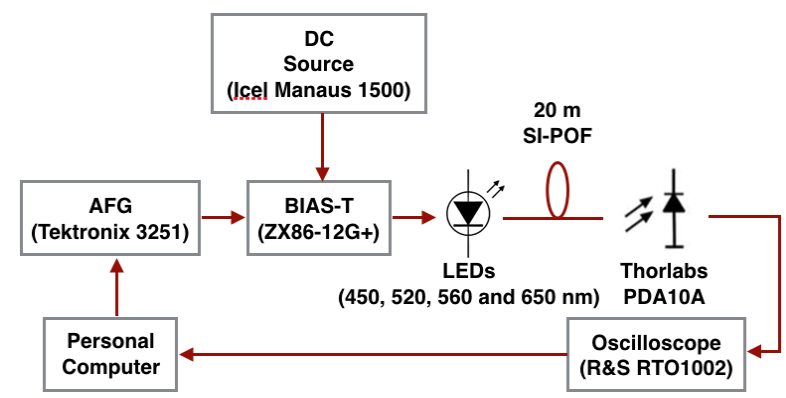

Figure 4 -Block diagram of the experimental setup.

All the transmissions were adjusted to have the same mean optical power at the photodetector for all operating wavelengths so that a fair comparison can be made between all emitters. All the baseband signals consisted of a $20 \mathrm{MHz}$ real-valued DMT signal with 491 useful subcarriers (IFFT size $=984$ considering DC and Nyquist null subcarriers). 16 samples per symbol are used as CP. The total DMT symbol duration is $25 \mu \mathrm{s}$, of each $24.6 \mu \mathrm{s}$ are the useful data and $0.4 \mu \mathrm{s}$ represent the guard interval. SNR, root-mean square error vector magnitude EVM
$\left(\mathrm{EVM}_{\mathrm{RMS}}\right)$ and BER per subcarrier are finally assessed over 1000 DMT symbols.

The DAC presents a 14-bit resolution, which generates a small quantization noise when compared to other sources of noise on the system. The DAC and ADC operate at $100 \mathrm{MSa} / \mathrm{s}$ and $400 \mathrm{MSa} / \mathrm{s}$ respectively, which is much higher than the optical transceiver bandwidth. Moreover, the input and output levels of DAC and ADC are properly chosen in order to minimize non-linear noise.

The transmitted power of each LED is normalized in order to deliver $-19.75 \mathrm{dBm}$ to the receiver.

\section{DMT TRANSMISSIONS RESULTS AND DISCUSSIONS}

As previously mentioned, the first step of the transmission is to send the probe signal to characterize the channel (SI-POF). The resulting received performance and spectra indicators are respectively shown in Figure 5 (top) and Figure 5 (bottom) for all the sources. Figure 5 (top) also shows an example of received signal in the time domain with 50 DMT symbols.

It can be seen from Figure 5 (bottom) that the channel acts as a low pass-filter and that for frequencies below 1.5 MHz some degradation can be seen due to the bias-t response.
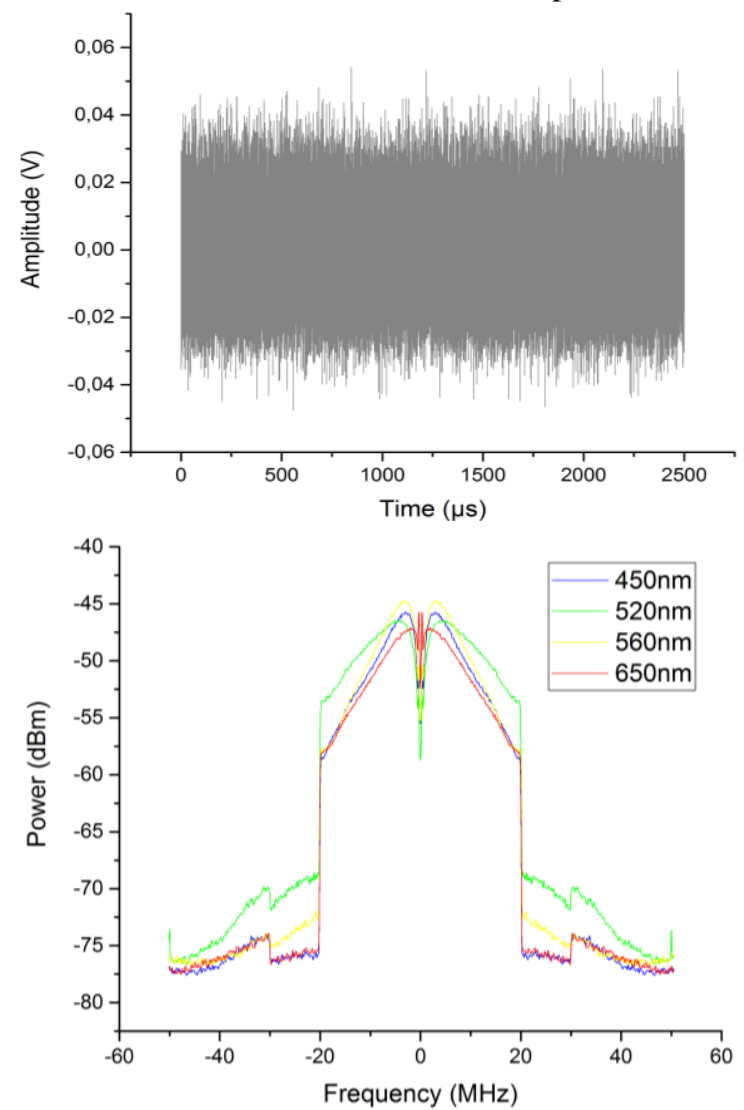

Figure 5 - Received time and frequency domain signal after 20 $\mathrm{m}$ of SI-POF, respectively.

This confirms the preliminary bandwidth assessment of Figure 2. The difference between the roll-offs in Figure 5 (bottom) for each optical source is due to the frequency response of the LEDs themselves. 


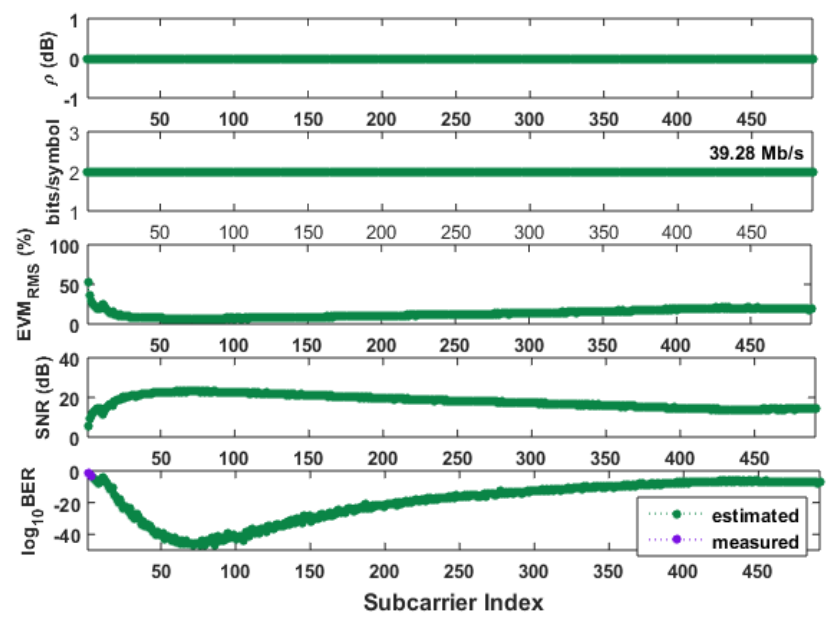

(a)

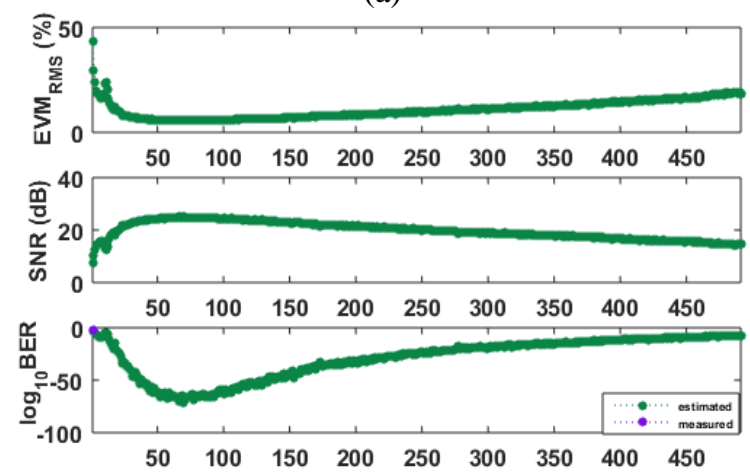

(b)
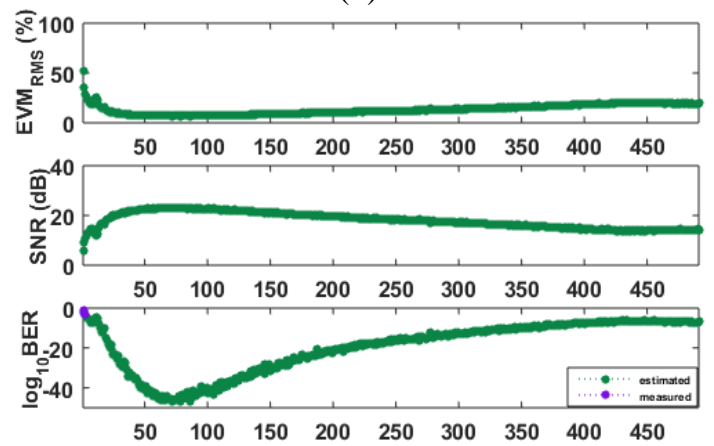

(c)

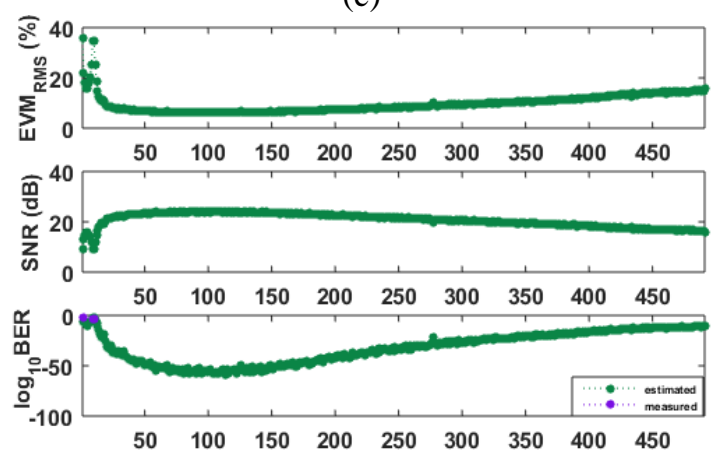

(d)

Figure 6 - DMT probe signal results with the LED at (a) $450 \mathrm{~nm}$, (b) $520 \mathrm{~nm}$, (c) $560 \mathrm{~nm}$ and (d) $650 \mathrm{~nm}$ showing the relative power coefficient, the number of bit per QAM.
In Figure 6, from top to bottom, are the relative power coefficient $(\rho)$ in $\mathrm{dB}$, the number of bits per QAM symbol $(\log 2(M)=2$ for $\mathrm{QPSK})$, the measured $\mathrm{EVM}_{\mathrm{RMS}}$, the SNR estimated from the EVM and the estimated BER for all subcarriers found with the probing signal for all the optical sources. For concision matters, only the relative power and the bit/symbol for $450 \mathrm{~nm}$ is shown. The others optical sources have the same behavior and follow the frequency profiles of Figure 5 (bottom). Note that, in Figure 6, the figure shows the first step of the modified Levin-Campello algorithm [15-16]. Then, all the sub-carriers use the same constellation, as explained in Section III.

Table 1 shows the average values for the EVM, SNR, estimated BER (E-BER) and measured BER (M-BER) from Figure 6.

Table 1: Summary of DMT probe signal results with LEDs.

\begin{tabular}{|l|c|c|c|c|}
\hline Optical Source & $450 \mathrm{~nm}$ & $520 \mathrm{~nm}$ & $560 \mathrm{~nm}$ & $650 \mathrm{~nm}$ \\
\hline EVM RMS (\%) & 9.29 & 10.95 & 13.32 & 9.66 \\
\hline SNR (dB) & 21.06 & 19.84 & 18.10 & 20.78 \\
\hline E-BER & $4.1010^{-5}$ & $2.3010^{-5}$ & $6.2010^{-5}$ & $1.4010^{-5}$ \\
\hline M-BER & $6.1010^{-5}$ & $2.4010^{-5}$ & $1.1010^{-5}$ & $3.5010^{-5}$ \\
\hline
\end{tabular}

The EVM values presented in this article are always measured along the sub-carriers with pilots. On the other hand, BER may be either estimated or measured. The estimation of BER is based on SNR, whereas the measurement of BER takes place after the application of the hard-decision on the receiver, by comparing these values to the transmitted ones on the pilots.

After sending the probe signal to evaluate the channel for each optical source $(450,520,560$ and $650 \mathrm{~nm})$, several transmissions were done for different pre-FEC targets BER $\left(10^{-}\right.$ $3,10^{-6}, 10^{-9}$ and $10^{-12}$ ) using the bit and power-loading algorithm. It can be seen in Figures 7 and 8 that the highest spectral efficiency achieved was $6.39 \mathrm{bits} / \mathrm{s} / \mathrm{Hz}$ for a pre-FEC target of $10^{-3}$ at $450 \mathrm{~nm}$ leading to a bit rate of $127 \mathrm{Mbps}$. After the demapping of the symbols, it is observed that the estimated BER from the SNR (green points) is in very good agreement with the measured BER (purple points) and that the target BER has been achieved. Indeed, a mean BER of $8.4 \cdot 10^{-4}$ is achieved. Besides, modulation levels as high as 128QAM (7 bit/symbol) were reached in 320 of 491 subcarriers $(65 \%)$.

Table 2 shows the average value of EVM, SNR, Estimated BER and Measured BER after the Levin-Campello algorithm is applied for all optical sources after $20 \mathrm{~m}$ of SI-POF. Also, it can be seen the data bit rate (DBR) and the achieved spectral efficiency (SE). 

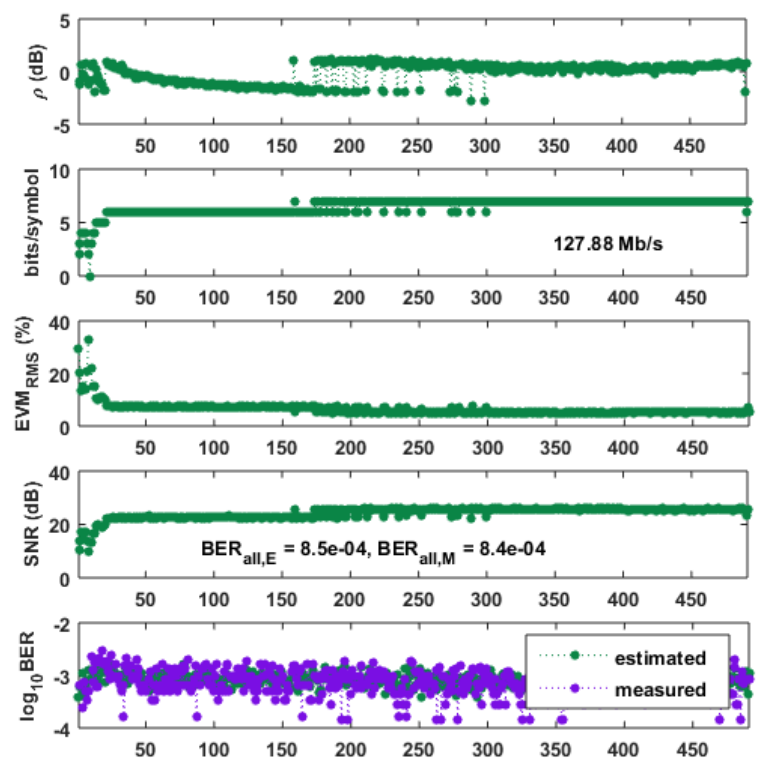

(a)

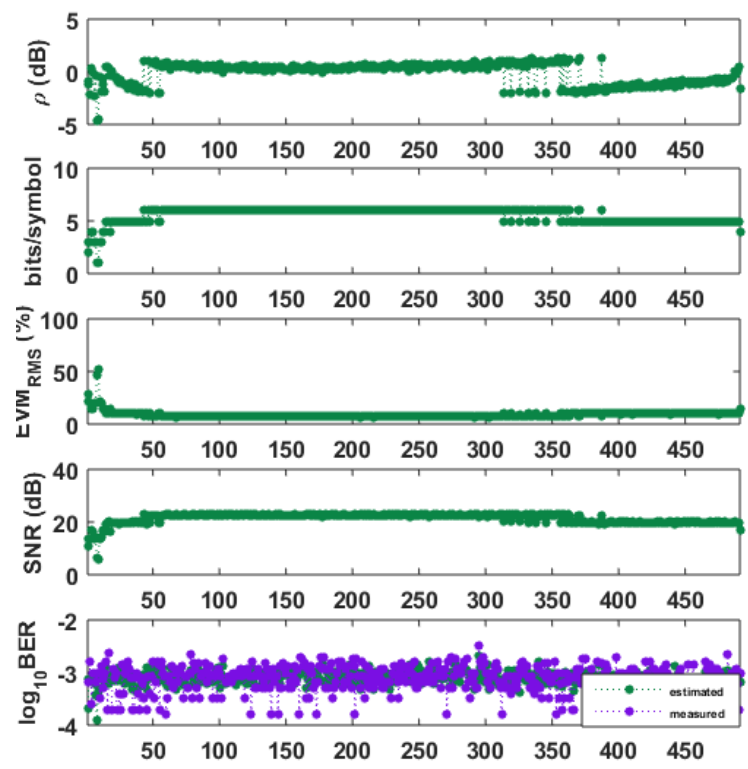

(b)

Figure 7 - Performance indicators per subcarrier after bit and power loading with the optical source at (a) $450 \mathrm{~nm}$ and (b) 520 $\mathrm{nm}$. In (a), the BER computation is performed for all the subcarriers, both measured (all,M) and estimated (all,E).

Since a target BER has been imposed, the overall data rate becomes constrained for each source. The source with highest rate is the $450 \mathrm{~nm} \mathrm{LED}$, with $127.9 \mathrm{Mbps}$. If a higher rate is used, there is further distortion and the target BER is not carried out.

As it was expected, the SE and DBR increases with the bandwidth (Figure 2). The M-BER is in agreement with the EBER evidencing that the algorithm makes the most of the SIPOF with the illumination LEDs.

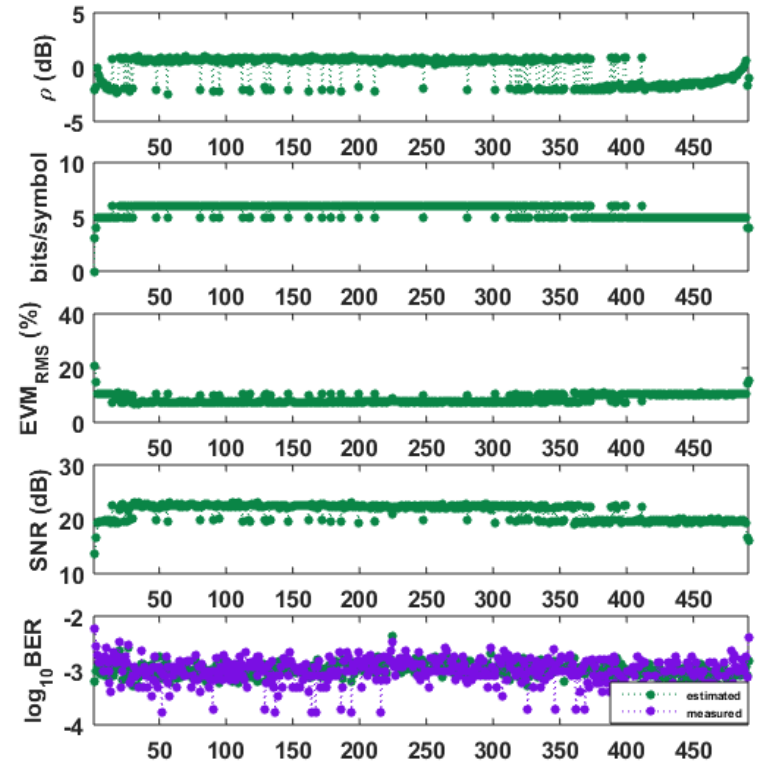

(a)

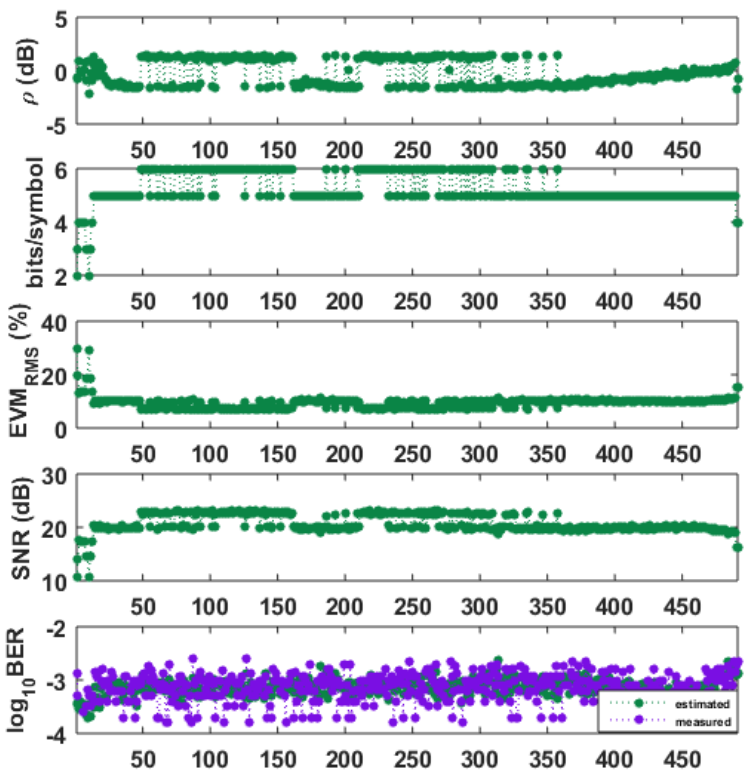

(b)

Figure 8 - Performance indicators per subcarrier after bit and power loading with the optical source at (c) $560 \mathrm{~nm}$ and (d) 650 nm after $20 \mathrm{~m}$ of SI-POF.

In Figure 9 it can be seen that higher than $3 \mathrm{bits} / \mathrm{s} / \mathrm{Hz}$ is obtained for target BER of $1 \cdot 10^{-12}$, which could allow avoiding the use of any error correcting scheme. Also, a close estimation of an aggregate bit rate that could be obtained in a WDM system can be performed. For pre-FEC target BER of $10^{-3}, 10^{-6}, 10^{-9}$ and $10^{-12}$ the transmission would allow 450, 357, 317 and 263 Mbps, respectively. 
Table 2: Average EVM, SNR, Estimated BER, Measured BER, Data Bit Rate and Spectral Efficiency for the optical sources at 450, 520, 560 and $650 \mathrm{~nm}$ after $20 \mathrm{~m}$ of SI-POF.

\begin{tabular}{|l|c|c|c|c|}
\hline Optical Source & $450 \mathrm{~nm}$ & $520 \mathrm{~nm}$ & $560 \mathrm{~nm}$ & $650 \mathrm{~nm}$ \\
\hline Energy (dB) & -0.12 & -0.12 & -0.17 & -0.18 \\
\hline Bits/Symbol & 6.51 & 5.57 & 5.62 & 5.31 \\
\hline EVM RMS (\%) & 6.37 & 8.77 & 8.62 & 9.37 \\
\hline SNR (dB) & 24.20 & 21.45 & 21.35 & 20.74 \\
\hline E BER & $8.5010^{-4}$ & $8.2010^{-4}$ & $1.1010^{-3}$ & $7.7010^{-4}$ \\
\hline M BER & $8.4010^{-4}$ & $8.4010^{-4}$ & $1.1010^{-3}$ & $8.2010^{-4}$ \\
\hline DBR (Mbps) & 127.88 & 112.22 & 110.28 & 104.20 \\
\hline SE (bits/s/Hz) & 6.39 & 5.61 & 5.51 & 5.21 \\
\hline
\end{tabular}

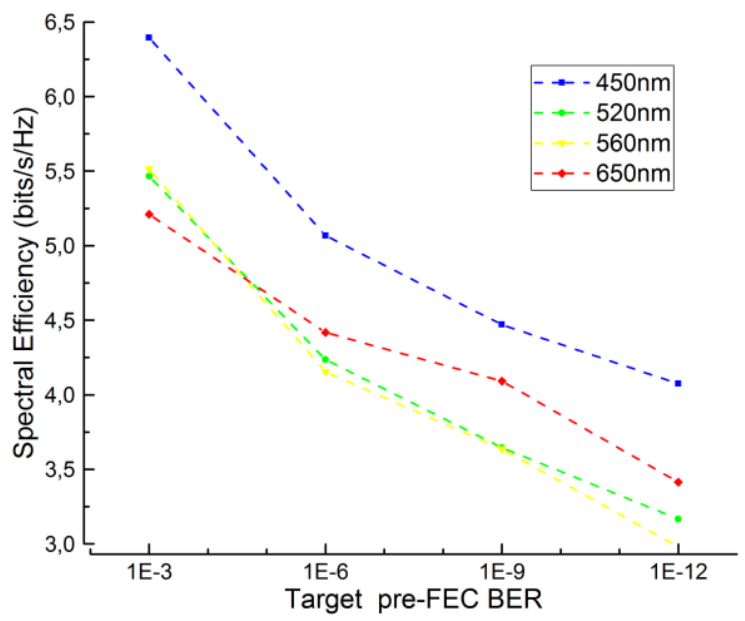

Figure 9 - Spectral efficiencies of the transmission in the blues, green, yellow and red channels for different pre-FEC target BER.

As can be noticed from Section II, each LED presents a different bandwidth. In spite of this, all the transmissions have been performed with $20 \mathrm{MHz}$, in order to provide a more uniform measurement framework. The computation of SE just considers the use of $20 \mathrm{MHz}$.

\section{CONCLUSION}

We have demonstrated DMT transmissions using a wavelength converted source emitting in the "yellow" wavelength experimentally demonstrated transmissions over $20 \mathrm{~m}$ of SIPOF using different illumination LEDs and a fluorescent optical fiber at $560 \mathrm{~nm}$, which has never been implemented in a WDM system. The results show that the use QAM plus DMT on these LEDs and on the fluorescent source is a good solution to increase the capacity of vehicle or in-home networks and thus that WDM systems with potentially high spectral efficiency could be developed. Up to $450 \mathrm{Mbps}$ of aggregate bit-rate for a pre-FEC target BER of $10^{-3}$ was demonstrated. Also, 3 bits $/ \mathrm{s} / \mathrm{Hz}$ could still be achieved while reducing the target BER to $10^{-12}$. Further research will focus on the transmission of all the signal at the same time by using a POF multiplexer and demultiplexer.

\section{REFERENCES}

[1] R. Kruglov, J. Vinogradov, O. Ziemann, S. Loquai and C. A. Bunge, "10.7-Gb/s Discrete Multitone Transmission Over 50-m SI-POF Based on WDM Technology," IEEE Photonics Technology Letters, vol. 24, no. 18, pp. 1632-1634, Sept.15, 2012, DOI: 10.1109/LPT.2012.2211583

[2] R. Kruglov, S. Loquai, J. Vinogradov, O. Ziemann, C. A. Bunge, G. Bruederl and U. Strauss, "10.7 Gb/s WDM Transmission over 10-m SIPOF with Discrete Multitone," in Proceedings of the Optical Fiber Communications Conference, pp. 4-5, Anaheim, USA, March 2016.

[3] R. Nazaretian and G. M. Molen, "Reducing Vehicle Weight and Improving Security by Using Plastic Optical Fiber," 2015 IEEE Vehicle Power and Propulsion Conference (VPPC), Montreal, QC, 2015, pp. 1-6.

[4] Y. Shi et al., "Plastic-optical-fiber-based in-home optical networks," IEEE Communications Magazine, vol. 52, no. 6, pp. 186-193, June 2014, DOI: 10.1109/MCOM.2014.6829963.

[5] R. Kruglov, J. Vinogradov, S. Loquai, O. Ziemann and C. A. Bunge, "Eye-safe data transmission of $1.25 \mathrm{Gbit} / \mathrm{s}$ over $100-\mathrm{m}$ SI-POF with four laser diodes in the visible range," OFC/NFOEC, Los Angeles, CA, 2012, pp. 1-3.

[6] O. Ziemann, L. Bartkiv, "POF-WDM, The Truth," 20th International POF Conference 2011, 2011 Bilbao.

[7] P. Pinzón, I. Garcilópez, and C. Vázquez, "Efficient Multiplexer/Demultiplexer for Visible WDM Transmission over SI-POF Technology," J. Lightwave Technologies, 33, 3711-3718 (2015), DOI: $10.1109 /$ JLT.2015.2455335.

[8] P. Bienias, G. Budzyń and E. Beres-Pawlik, "WDM for application in passive POF LAN networks," 2016 18th International Conference on Transparent Optical Networks (ICTON), Trento, 2016, pp. 1-3.

[9] M. Joncic, R. Kruglov, M. Haupt, R. Caspary, J. Vinogradov and U. H. P. Fischer, "Four-Channel WDM Transmission Over 50-m SI-POF at 14.77 Gb/s Using DMT Modulation," IEEE Photonics Technology Letters, vol. 26, n. 13, pp. 1328-1331, July 2014.

[10] B. Charbonnier et. al., "Capacity optimisation for optical links using DMT modulation, an application to POF," in Proceedings of the European Conference on Optical Communication, v. 3, pp. 221-222, Brussels, Belgium, Sept. 2008.

[11] X. Li et al., "11 Gb/s WDM Transmission for optical links using DMT modulation, an application to POF," in Proceedings of the Optical Fiber Communications Conference, pp. 1-3 Anaheim, USA, March 2016.

[12] R. Caspary et al., "High speed WDM transmission on standard polymer optical fibers," 2015 17th International Conference on Transparent Optical Networks (ICTON), Budapest, 2015, pp. 1-4. DOI: 10.1109/ICTON.2015.7193418

[13] R. M. Ribeiro, V. N. H. Silva, A. P. L. Barbero, C. M. Alves and C. R. L. Rodrigues, "Fast wavelength conversion to generate $560 \mathrm{~nm}$ fluorescence for data transmission in polymer optical fibers," Electronics Letters, vol. 51, n. 2, pp. 168-170, Jan. 2015, DOI: 10.1049/el.2014.3871.

[14] J. Armstrong, "OFDM for Optical Communications," Journal of Lightwave Technology, vol. 27, no. 3, pp. 189-204, Feb.1, 2009, DOI: $10.1109 /$ JLT.2008.2010061

[15] L. A. Neto et al., "High bit rate burst mode optical OFDM for next generation passive optical networks," 36th European Conference and Exhibition on Optical Communication, Torino, 2010, pp. 1-3. doi: 10.1109/ECOC.2010.5621162

[16] L. Anet Neto, Étude des Pontentialités des Techniques de Modulation Multiporteuse pour les Futurs Réseaux D'Accès Optique WDM et TDM $P O N$, Doctorate Thesis, Université de Limoges, France, 2012.

[17] B. Cardiff et al., "On Bit and Power Loading for OFDM over SI-POF," Journal of Lightwave Technology, vol. 29, n. 10, pp. 1547-1554, May 2011, DOI: 10.1109/JLT.2013.2290811. 


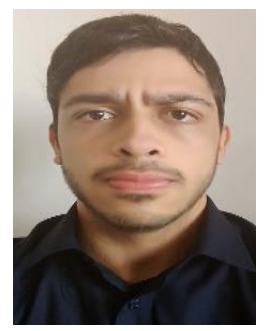

Flávio André Nogueira Sampaio, was born in Rio de Janeiro, Brazil. $\mathrm{He}$ is an undergraduate student at Fluminense Federal University (UFF) in Niterói, Brazil. $\mathrm{He}$ is currently involved in the research areas of Orthogonal Frequency Division Multiplex, Radio Over Fiber, Plastic Optical Fiber communications and nonlinear effects of Optical sources at the Optical Communications Laboratory (LACOP) where he is a scientific initiation scholarship holder.

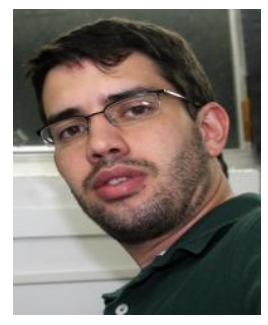

Vinicius Nunes Henrique Silva was born in Rio de Janeiro, Brazil in 1979. He received the Telecommunications Engineer degree and M.Sc. degree in Photonic Systems from Universidade Federal Fluminense, Rio de Janeiro, Brazil in 2006 and 2009 respectively. He was awarded with his Ph.D. degree by the École Nationale Supérieure Mines-Télécom Atlantique Bretagne-Pays de la Loire in 2013 after working as an R\&D engineer at Eyes3Shut, Brest, France. His doctoral work focused on Liquid Crystal Shutter for 3D Cinema and Photonics Applications. He is currently working as a professor and researcher at Universidade Federal Fluminense where he mainly works with applied optics/photonics for sensors and telecommunication. His research interests include optical instrumentation, optical modulation schemes and digital signal processing techniques.

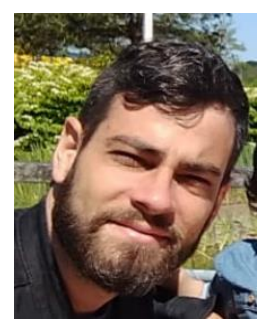

Luiz Anet Neto was born in Rio de Janeiro, Brazil in 1984. He received the Telecommunications Engineer degree and M.Sc. degree in Photonic Systems from Universidade Federal Fluminense, Rio de Janeiro, Brazil in 2007 and 2009 respectively. He also holds a M.Sc. degree in Physics and Photonics from Ecole Nationale Supérieure de Télécommunications de Bretagne (ENSTB), Brest, France (2009). He was awarded with his Ph.D. degree by the Université de Limoges in 2012 after working as an R\&D engineer at Orange Labs, Lannion, France. His doctoral work focused on DMT transmissions over PON. From 2013 to 2015, he worked as a postdoctoral researcher at FOTON - ENSSAT. At the time, he focused his works on optically filtered DIM/DD solutions for next generation PON and on tunable silicon-photonics based filters for optical signal processing purposes. He is currently working as a research engineer at Orange Labs where he mainly investigates functional split based radio access optical network interfaces for the following generation of mobile systems. His research interests include advanced optical modulation schemes, digital signal processing techniques, $\mathrm{WDM}(\mathrm{A}) / \mathrm{TDM}(\mathrm{A}) / \mathrm{FDM}(\mathrm{A})$ solutions for next generation passive optical networks, alloptical signal processing, the optical xhaul and the impacts of virtualization and software defined networks. He is also a big enthusiast of raspberrypi projects.

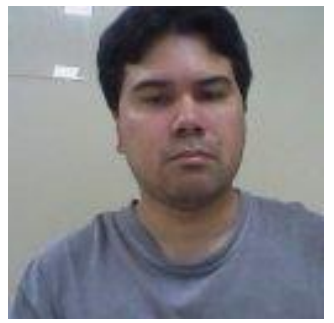

Tadeu Ferreira is D.Sc. in Electrical Engineering (UFRJ, 2009). He is an adjunct professor at Fluminense Federal University (UFF) in Niterói, Brazil. Previously, he was a technical high-school teacher at CEFET UnED Nova Iguaçu and a post-doc fellow at UFRJ. In 2015, he was awarded 2nd place at Excellency in Teaching Award at UFF. This is his 9th journal article. So far, he has advised 7 M.Sc. candidates.

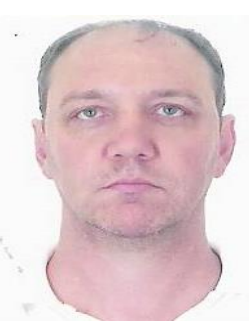

Professor Andrés Pablo Lopez Barbero was born in Montevideo, Uruguay in 1966. He receiveid the B. Sc degree in Telecommunications Engineering from the Federal Fluminense University, Niteroi, Rio de Janeiro, Brazil, in 1988. $\mathrm{He}$ received his M.Sc degree in Electronic Engineering from the Technological Institute of Aeronautics ITA, in 1991, and his Ph.D degree in Electrical Engineering from the State University of Campinas (Unicamp), in 2000. Since 1989 he is professor at the Telecommunications Department of the Federal Fluminense University and his research interests include Optical Fiber communicatios, and optical fiber sensing based on Bragg Gratings (FBG and LPG).

Professor Ricardo Marques Ribeiro has DSc. in Physics (1995). From 1996 to 2005 he worked with photorefractive fibres, external cavity mode-locked lasers (British Telecom Research Laboratories - UK) and optoelectronics applied to instrumentation and sensing. Since 2006 he is Professor of applied Electromagnetism and Photonics Researcher on the Universidade Federal Fluminense. He has more than 80 internationally published papers and many technological developments. From Aug/2012 to Nov/2013 he joined the Optics Department of Telecom Bretagne in Brest/France for a Post-Doc stage. His main interests and R\&D efforts are on Fibre-Optic Devices for Telecommunications, all-optical signal processing and Polymer Optical Fibres. 